\title{
THEORETICAL WAVELENGTH DEPENDENCE OF

\author{
POLARIZATION IN EARLY-TYPE STARS
}

\author{
BERNHARD M. HAISCH and JOSEPH P. CASSINELLI \\ Washburn Observatory, University of Wisconsin, Madison, Wis., U.S.A.
}

\begin{abstract}
We have examined the theoretical wavelength dependence of linear polarization produced by electron scattering and modified by an absorptive opacity in the extended, distorted atmospheres of Be and Wolf-Rayet stars. A model atmosphere representing a Wolf-Rayet star recently calculated by Cassinelli and Hartmann was used. This model takes into consideration the subsonic portions of a radial, steady-state flow together with the requirement of radiative equilibrium. The model was then scaled down in temperature to approximate the cooler Be stars, again assuming radiative equilibrium. There were four models altogether, one WR star, and three Be models. All the atmospheres are quite extended by radiation pressure.
\end{abstract}

We then wrote the equation of transfer in spherical coordinates for $I_{L}$ and $I_{R}$, the intensities in the radial and tangential planes of polarization, took the zeroth and first moments of this equation, and combined them into a second order equation to be solved numerically by iterating on a variable Eddington factor, $K / J$. The results showed polarization as high as $\mathbf{7 0 \%}$ at the limbs of the atmospheres. However, in order to produce polarization in the net flux, an asymmetry must exist in the shape of the star. We therefore constructed disc models by simply truncating a spherical atmosphere, and Roche models, in which equipotential surfaces are assumed also to be surfaces of equal source function with a modification to account for the forward peaking of the radiation field. The polarization in the net flux was then calculated for all four model atmospheres for each of these two distorted shapes.

For the disc models we find that the polarization increases from about $\frac{1}{3} \%$ at $1 \mu^{-1}$ to about $1 \%$ near $2.5 \mu^{-1}$, with drops across the absorption edges of $\mathrm{H}$ and $\mathrm{He}$. The reduction in polarization across these edges is due to the absorption of polarized light and subsequent thermal re-emission of unpolarized light. The overall rise of the polarization toward shorter wavelength is due to the steeper gradient of the Planck functions at higher frequencies. The strength of the polarization decrease across the absorption edges, as well as the overall strength of the polarization, changes moderately from the hottest to the coolest models.

For the Roche models, the behavior is qualitatively similar, but the overall strength of the polarization is markedly reduced $(0.2$ to $0.3 \%)$. In all the models, the net polarization is perpendicular to the polar axis. None of the models predicts an upturn in the polarization longward of the Paschen edge as is cited in some wide-band polarimetric observations. The drop in the polarization across the Balmer limit is severe only for the coolest models. The slope between the Balmer and Paschen edges agrees well with observations. However, it is found that only a rather flattened disc having a ratio of thickness to radial depth of about $\frac{1}{3}$ can provide polarization as high as is observed. 
The energy distributions of the Roche models show Balmer continuous emission, except for the very coolest model which shows a Balmer discontinuity of zero. This is due to the extreme geometrical extensions of the atmospheres.

\section{DISCUSSION}

Hummer: Are your models based on the LTE assumption? If so, I would doubt your Balmer discontinuities in emission. In a large number of extended spherical, non-LTE model atmospheres computed by Kunasz, Mihalas and myself (Astrophys. J., in press), the Balmer jumps are always in absorption by roughly 0.05 mag. 